\title{
Programas de prevención de obesidad infantil en México: una revisión sistemática PICO
}

\section{Prevention programs of child obesity in Mexico: A PICO systematic review}

\author{
Alejandro Pérez Ortiz, Mayaro Ortega Luyando \\ y Adriana Amaya Hernández
}

\author{
Universidad Nacional Autónoma de México ${ }^{1}$
}

Autor para correspondencia: Alejandro Pérez Ortiz, aleixen_psi10@yahoo.com.mx.

\begin{abstract}
RESUMEN
El sobrepeso y obesidad infantil en México han alcanzado proporciones epidémicas, afectando hasta $33.2 \%$ de la población. Identificar qué tipo de programas de prevención se han llevado a cabo y qué profesionales de la salud intervienen en su aplicación es una tarea necesaria para identificar si estos han sido efectivos. Por lo tanto, los objetivos de la presente revisión sistemática fueron: a) identificar los programas de prevención de obesidad infantil en México, $b$ ) analizar su metodología e indicadores de cambio utilizados para evaluar la efectividad del programa y c) identificar asimismo la teoría psicológica que utiliza y las funciones que desempeña el psicólogo en dichos programas, para lo cual se realizó una búsqueda sistemática bajo la metodología PICO en seis bases de datos: Scopus, Scielo, Pubmed, Web of Science, Redalyc y Tesiunam. Se incluyen los estudios realizados en población escolar mexicana de 5 a 11 años en el periodo de 2008 a 2018. Se encontraron 3,502 artículos, y tras aplicar los criterios de inclusión y exclusión se analizaron seis estudios. Los hallazgos señalan que el índice de masa corporal es el parámetro más empleado para evaluar la efectividad de un programa, y que las perspectivas conductual e interconductual suelen ser las más utilizadas para el diseño, aplicación y evaluación de los programas de prevención cuando se trata de estudios realizados por psicólogos, lo cual suele estar ausente en estudios a cargo de otros profesionales de la salud.
\end{abstract}

Palabras clave: Sobrepeso; Infancia; Promoción de la salud; Salud pública; Estado del arte.

\begin{abstract}
Childhood overweight and obesity in Mexico have reached epidemic proportions affecting up to $33.2 \%$ of the population. Identifying the type of prevention programs being carried out and which health professionals are involved in their application is necessary to assess their effectiveness. Thus, the objectives of the present systematic review were: a) to identify programs for the prevention of childhood obesity in Mexico, b) to analyze their methodology and indicators of change in order to assess their effectiveness and c) to identify associated psychological theories and the contributions of psychologists in such prevention programs. The search was carried out following the PICO methodology in six databases: Scopus, Scielo, Pubmed, Web of Science, Redalyc and TesiunAM. Eligible studies were those conducted with Mexican elementary school children (5 to 11 years old), and published between 2008 and 2018. A total of 3502 articles were located, after
\end{abstract}

\footnotetext{
${ }^{1}$ Grupo de Investigación en Nutrición, División de Investigación y Posgrado, Facultad de Estudios Superiores Iztacala, Av. De los Barrios s/n, Col. Los Reyes Iztacala, 54090 Tlalnepantla, Edo. de México, México, tel. 555 623-13-33 ext. 39737, correos electrónicos: aleixen_psi10@, yahoo.com.mx, psic.mayaro@gmail.com y amayahad@gmail.com.
} 
applying the inclusion and exclusion criteria and six articles were finally analyzed in depth. Body mass index was the recurrent indicator of change to assess the effectiveness of the programs. Interbehaviorism and behaviorism were the most used perspectives to design, apply, and assess preventive programs, when the studies were performed by psychologists. These approaches are not usually adopted in studies conducted by other health professionals.

Keywords: Overweight; Infancy; Health promotion; Public health; State of art.

Recibido: $10 / 10 / 2019$

Aceptado: 12/05/2020

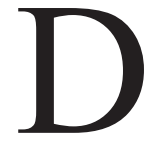
e acuerdo con la Organización Mundial de la Salud (OMS) (2018), el sobrepeso y la obesidad se definen como una acumulación anormal o excesiva de grasa que puede ser perjudicial para la salud; ambas condiciones pueden identificarse mediante el cálculo del índice de masa corporal (IMC en lo sucesivo), que es una medida sensible para detectar el estado nutricional de una persona. Se ha reconocido que la obesidad es una enfermedad crónica de origen multicausal (interacción entre variables biopsicosociales) que puede desarrollarse en la infancia o adolescencia (Muñoz y Arango, 2017; Puente, 2008; Rivera, Hernández, Aguilar, Vadillo y Murayama, 2012; Torrico, Santín, Andrés, Álvarez y López, 2008).

Se estima que hasta 2016 había 124 millones de personas de entre 5 y 19 años con obesidad en todo el mundo, y que 213 millones sufrían sobrepeso. En el continente americano los países que presentan los índices más altos de obesidad entre la población escolar de 5 a 10 años son México, Estados Unidos y Canadá (34.4, 34.3 y 32.8\%, respectivamente); mientras que en el rango de 11 a 19 años están Bolivia, México, nuevamente, y Estados Unidos (42.7, 35 y $34.5 \%$, en cada caso) (OMS, 2018; Organización Panamericana de la Salud [OPS], 2014). De esta forma, México es una de las naciones con los índices más altos de obesidad en dichas poblaciones. Muestra de ello es la Encuesta Nacional de Salud y Nutrición-Medio Camino (ENSANUT-MC) (Shamah, Cuevas, Rivera y Hernández, 2016), que reportó una prevalencia de sobrepeso y obesidad de $33.2 \%$ entre los niños de 5 a 11 años.

La población infantil que padece obesidad puede sufrir complicaciones de salud incluso en la edad adulta, entre las que se encuentran las siguientes: depresión, ansiedad, insatisfacción corporal, baja autoestima, mayor probabilidad de muerte, desarrollo de discapacidades prematuras en la etapa adulta, diabetes, hipertensión, enfermedades cardiovasculares, trastornos del aparato locomotor y ciertos tipos de cáncer (mama, colon y endometrio) (Mercado y Vilchis, 2013; OMS, 2018). Ante dicha situación, se ha propuesto que prevenir la enfermedad es la medida óptima para hacer frente a este problema debido a que hace posible promover la salud entre la población mediante la creación de políticas y programas de salud, y con ello controlar o retrasar las complicaciones de la enfermedad a mediano y largo plazo, y disminuir la inversión en el sector salud por las complicaciones derivadas de la obesidad (Franco, 2012; Instituto Mexicano para la Competitividad, 2015; Lizaraso, 2012; OMS, 2016; OPS, s.f.; Ortega, 2014; Secretaría de Salud, 2018). Trabajar con la población infantil permitirá promover estilos de vida más saludables, ya que en la niñez se aprenden e imitan las conductas y prácticas de los padres, familiares y pares con los que interactúa el menor. Desde la perspectiva de la psicología conductual y cognitivo-conductual es posible modificar los patrones de conducta y estilos de vida no saludables en básicamente cualquier condición de salud-enfermedad mediante programas de intervención emanados de las técnicas de modificación de conducta (Mercado y Vilchis, 2013; Reynoso, 2014; Reynoso y Seligson, 2005).

En consecuencia, los objetivos de la presente investigación fueron, a saber: a) identificar los programas de prevención de la obesidad infantil que se desarrollan en México; $b$ ) analizar su metodología e indicadores de cambio utilizados para evaluar la efectividad de la intervención, y c) identificar la teoría psicológica de los psicólogos y las funciones que desempeñan en dichos programas. 


\section{MÉTODO}

\section{Estrategia de búsqueda}

Se realizó una búsqueda sistemática mediante la estrategia PICO, dado que el objetivo de este estudio no fue comparar dos tipos de intervenciones, para lo cual se ajustó la búsqueda al acrónimo PIO (Jensen, 2018; Landa y Arredondo, 2014; Rivas y Talavera, 2012).

\section{Procedimiento}

Se efectuó la búsqueda a lo largo de dos meses en las bases de datos Scopus, Scielo, Pubmed,
Web of Science, RedalyC y Tesiunam. En las primeras cinco bases se emplearon las palabras clave que se muestran en la Tabla 1 en diferentes combinaciones, mientras que en la base de datos TesiUNAM se emplearon las combinaciones "obesidad infantil $A N D$ psicología, y "obesidad infantil $A N D$ intervención" puesto que dicha base no cuenta con un sistema de búsqueda que permita seguir la metodología PIO. Para delimitar los resultados de la búsqueda se empleó el operador booleano $A N D$ y se aplicaron los filtros a las bases de datos establecidos previamente (país, idioma, rango de fecha, tipo de documento y área de investigación).

Tabla 1. Palabras clave en inglés y español de los componentes de la búsqueda PIO.

\begin{tabular}{|l|l|l|}
\hline \multicolumn{1}{|c|}{ P } & \multicolumn{1}{c|}{ I } & \multicolumn{1}{c|}{ O } \\
\hline Mexican obese children & Psychological treatment & Body Mass Index \\
\hline Mexican children with obesity & Interdisciplinary treatment & Eating behaviors \\
\hline Mexican children with overweight & Multidisciplinary treatment & Physical activity \\
\hline \multicolumn{3}{|c|}{} \\
\hline Niños mexicanos obesos & Intervención psicológica & Índice de masa corporal \\
\hline Niños mexicanos con obesidad & Intervención interdisciplinar & Conducta alimentaria \\
\hline Niños mexicanos con sobrepeso & Intervención multidisciplinar & Actividad física \\
\hline
\end{tabular}

\section{Selección de artículos}

Para la selección de estudios se propusieron los siguientes criterios de inclusión y exclusión:

Criterios de inclusión: a) intervenciones dirigidas a prevenir la obesidad infantil en niños con un rango de edad de 5 a 11 años; $b$ ) intervenciones psicológicas, interdisciplinares o multidisciplinares con componentes psicológicos; c) estudios de enfoque cuantitativo o cualitativo; $d$ ) publicaciones en idioma español e inglés, y e) publicaciones de los últimos diez años.

Criterios de exclusión: a) publicaciones en idiomas diferentes al español e inglés; b) revisiones sistemáticas, análisis teóricos, capítulos de libros o propuestas de intervención; c) trabajos realizados en países diferentes a México, y d) que los participantes estuviesen fuera del rango de edad seleccionado.

\section{Ejes de análisis de los artículos}

Para responder a los objetivos de la investigación se extrajo de cada artículo la siguiente información: autor(es), año, muestra, instrumentos, modelo teórico, participación de padres, abordaje multidisciplinar, duración, indicadores de cambio y seguimiento.

\section{RESULTADOS}

Para responder a cada objetivo planteado, y retomando los ejes de análisis del contenido de los artículos, se presentan los resultados en tres secciones:

\section{Programas de prevención de obesidad infantil en México}

La búsqueda arrojó 3,444 artículos, de los que, al ser analizados bajo los criterios de inclusión y 
exclusión, solo seis fueron seleccionados para su revisión. En la Figura 1 se muestra el diagrama de flujo del trabajo hecho, y en la Tabla 2 las ca- racterísticas metodológicas de los programas de prevención.

Figura 1. Diagrama de flujo de la búsqueda y selección de programas de prevención de obesidad infantil en población mexicana.

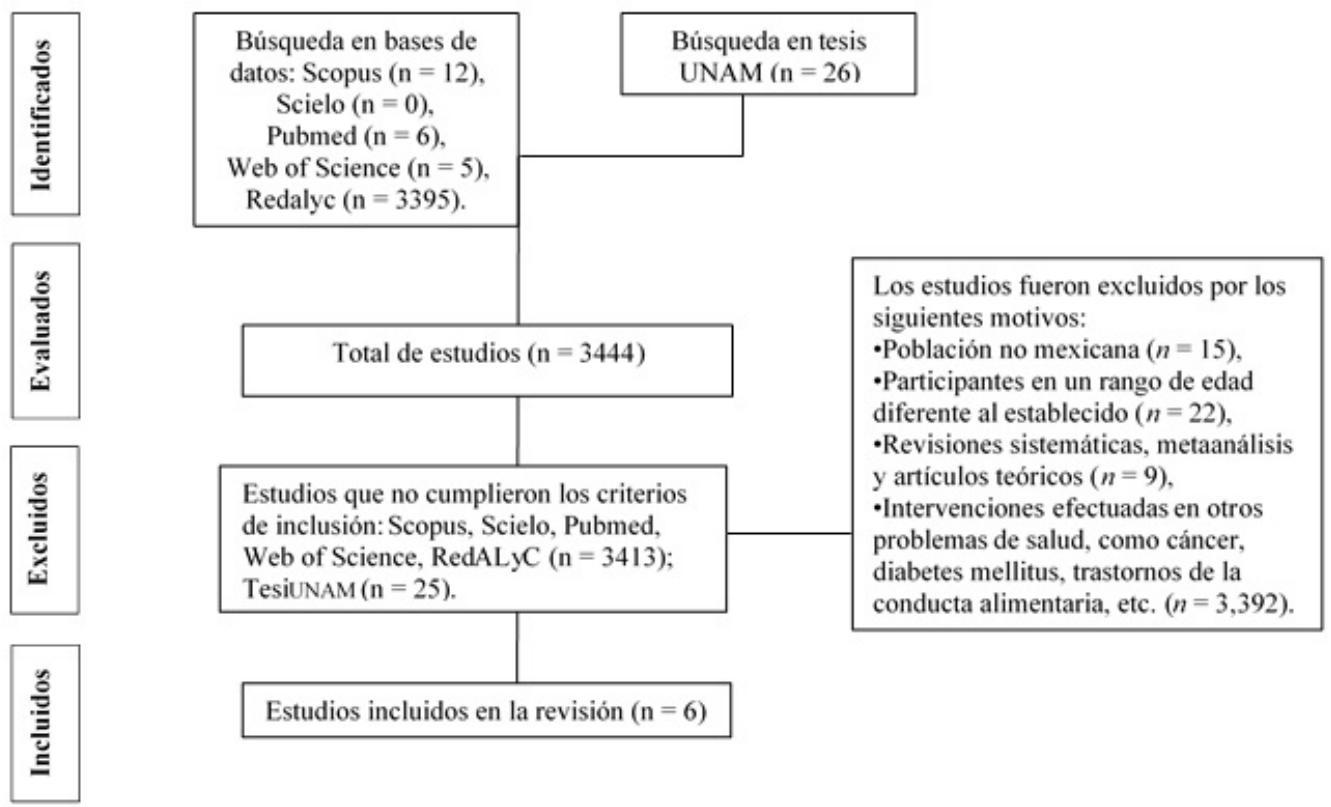

\begin{tabular}{|c|c|c|c|c|c|c|c|c|}
\hline $\begin{array}{l}\text { Autor } \\
\text { y año }\end{array}$ & Muestra (n) & Instrumentos & $\begin{array}{c}\text { Modelo } \\
\text { teórico }\end{array}$ & $\begin{array}{c}\text { Participación } \\
\text { de padres }\end{array}$ & $\begin{array}{c}\text { Abordaje } \\
\text { multidisciplinar }\end{array}$ & Duración & $\begin{array}{c}\text { Indicadores } \\
\text { de cambio }\end{array}$ & Seguimiento \\
\hline $\begin{array}{l}\text { Alvirde } \\
\text { et al. } \\
(2013)\end{array}$ & $\begin{array}{l}n=2,682 \\
\text { Grupo experimental: } \\
(n=1,927), \\
\text { edad }(M=9.0, D . E .=1.7), \\
\text { IMC }(M=18.6, D . E .=3.37) \\
\text { Grupo control: } \\
(n=755), \text { edad } \\
(M=9.1, \text { D.E. }=1.7), \\
\text { IMC }(M=18.3, \text { D.E. }=3.0) .\end{array}$ & & & & & Tres años. & $\begin{array}{l}\text { IMC, orienta- } \\
\text { ción nutricio- } \\
\text { nal, actividad } \\
\text { física e infor- } \\
\text { mación sobre } \\
\text { estilos de vida } \\
\text { saludable. }\end{array}$ & \\
\hline $\begin{array}{l}\text { Cortés } \\
\text { et al. } \\
(2009)\end{array}$ & $\begin{array}{l}n=116 \\
\text { Alumnos de } 2^{\circ}, 4^{\circ} \text { y } 6^{\circ} \\
\text { grados de primaria, con } \\
\text { rango de edad de } 7 \text { a } 13 \\
\text { años. }\end{array}$ & & $\begin{array}{l}\text { Intercon- } \\
\text { ductual }\end{array}$ & & & $\begin{array}{l}20 \text { días, en } \\
\text { sesiones de } \\
50 \text { minutos. }\end{array}$ & $\begin{array}{l}\text { Preferencia } \\
\text { y selección } \\
\text { por alimentos } \\
\text { saludables. }\end{array}$ & \\
\hline $\begin{array}{l}\text { García } \\
(2008)\end{array}$ & $\begin{array}{l}n=33 \\
16 \text { padres, y } 17 \text { niños con } \\
\text { rango de edad de } 5 \text { a } 11 \\
\text { años. }\end{array}$ & $\begin{array}{l}\text { Test de Actitud } \\
\text { en Contra de } \\
\text { la obesidad } \\
\text { (AFAT). }\end{array}$ & & $\begin{array}{l}\text { Modificar su } \\
\text { actitud hacia la } \\
\text { obesidad. }\end{array}$ & & $\begin{array}{l}\text { Cinco } \\
\text { sesiones de } \\
\text { dos horas } \\
\text { cada una, } \\
\text { impartidas } \\
\text { una vez por } \\
\text { semana. }\end{array}$ & IMC & \\
\hline
\end{tabular}

Continúa...

Instituto de Investigaciones Psicológicas - Universidad Veracruzana ISSN impreso: 1405-1109
Psicología y Salud, Vol. 31, Núm. 2: 169-177, julio-diciembre de 2021 https://doi.org/10.25009/pys.v31i2.2686 


\begin{tabular}{|c|c|c|c|c|c|c|c|c|}
\hline $\begin{array}{l}\text { González } \\
\text { et al. } \\
(2014)\end{array}$ & $\begin{array}{l}n=60 \\
30 \text { en el grupo experi- } \\
\text { mental y } 30 \text { en el grupo } \\
\text { control; rango de edad de } \\
\text { los niños, de } 6 \text { a } 12 \text { años, } \\
\text { IMC (M }=21.67 \text {, } \\
D . E .=3.84) . \text { Padres y } \\
\text { niños con sobrepeso u } \\
\text { obesidad }\end{array}$ & & & $\begin{array}{l}\text { Estar en } \\
\text { régimen } \\
\text { alimenticio (no } \\
\text { especifica plan } \\
\text { de alimenta- } \\
\text { ción). }\end{array}$ & & Seis meses. & IMC & \\
\hline $\begin{array}{l}\text { Rivera } \\
\text { et al. } \\
(2010)\end{array}$ & & Ex profeso & & $\begin{array}{l}\text { Modificar la } \\
\text { preparación de } \\
\text { los alimentos } \\
\text { (hervirlos } \\
\text { o asarlos) y } \\
\text { disminuir el } \\
\text { consumo de } \\
\text { azúcar. }\end{array}$ & & Un año. & IMC & \\
\hline
\end{tabular}

Nota $: \mathrm{IMC}=$ índice de masa corporal; AFAT = Test de Actitud en Contra de la obesidad (por sus siglas en inglés) (Lewis, Cash, Jacobi y Bubb-Lewis, 1997); cf. García, 2008). Los estudios difieren en la información de sus muestras, lo que impide homogeneizarlas.

\section{Metodología e indicadores de cambio utilizados para evaluar la efectividad de la intervención}

\section{Muestra}

La muestra osciló entre 33 y 2,682 participantes $\left(M_{e}=116\right)$. En todas las intervenciones participaron niños de ambos sexos; en cuatro de ellas se incluyó a los padres de familia, siendo las madres las que participaron en su mayoría. El rango del número de padres que participaron osciló entre 4 y $62\left(M_{e}=16\right)$; cinco de las intervenciones se realizaron en población infantil que padecía sobrepeso u obesidad, teniendo como objetivo disminuir el IMC, y un estudio se hizo en población con un IMC normal.

\section{Abordaje multidisciplinar}

Solamente en un estudio se reportó un enfoque multidisciplinar que involucró a enfermeras, tra- bajadores sociales, nutriólogos y educadores físicos. En contraste, tres estudios fueron exclusivamente nutriológicos y dos psicológicos.

\section{Participación de padres}

Entre las actividades que efectuaron los padres se encuentran, a saber: estar en un régimen alimenticio saludable, al igual que sus hijos (González, Castañeda, López, Brito y Sabag, 2014); modificar la preparación de los alimentos (hervirlos o asarlos en vez de freírlos); disminuir el consumo de azúcar en la familia (Rivera, Priego y Córdova, 2010); modificar su actitud hacia la obesidad (García, 2008), y leer folletos informativos sobre cómo preparar desayunos saludables para sus hijos (Safdie et al., 2013). Ningún estudio reportó haber llevado a cabo sesiones con padres e hijos a la vez, ni tampoco integrar una fase de evaluación que evidenciara la adquisición del conocimiento para llevarlo a la práctica. 


\section{Instrumentos}

Dos de los estudios crearon sus propios instrumentos para evaluar la percepción de la imagen corporal, los conocimientos sobre alimentación que tenían los niños y el consumo de agua. En otra investigación se empleó el Test de Actitud en Contra de la Obesidad (AFAT) de Lewis, Cash, Jacobi y Bubb-Lewis (1997), el cual posee un coeficiente alfa de Cronbach adecuado (.88) y que fue validado en población mexicana previamente a la intervención. Los demás estudios no reportaron el uso de algún instrumento.

\section{Duración de las intervenciones}

La intervención de menor duración consistió de cinco sesiones de dos horas cada una, impartidas una vez por semana (García, 2008); la segunda en ese orden duró veinte días hábiles en sesiones de 50 minutos (Cortés, López, Alarcón y Torres, 2009); la tercera duró seis meses (González et al., 2014); la cuarta siete meses (Safdie et al., 2013), y la quinta un año (Rivera et al., 2010); la intervención con la máxima duración fue de tres años (Alvirde, Rodríguez, Henao, Gómez y Aguilar, 2013).

\section{Indicadores de cambio}

Por un lado, cinco de las seis intervenciones retomaron el IMC como indicador para determinar si la intervención tuvo efectos en los participantes, aunque los resultados de solo tres de los cinco programas fueron estadísticamente significativos (Alvirde et al., 2013; García, 2008; González et al., 2014). Por otro lado, la investigación de Cortés et al. (2009) retomó la preferencia y selección de alimentos saludables, con resultados estadísticamente significativos únicamente en la preferencia de alimentos.

\section{Seguimiento}

Solamente el estudio de Safdie et al. (2013) llevó a cabo una fase de seguimiento, en la que mantuvieron las mismas actividades hechas durante la intervención (orientación nutricional, actividad físi- ca e información sobre estilos de vida saludable), aunque también incorporaron algunas tareas nuevas, tales como prohibir el consumo de alimentos altos en grasa y reducir la venta de dulces dentro de las escuelas primarias. Dicha fase tuvo una duración de ocho meses, un mes más de lo que duró la intervención. Por otro lado, solo una de las intervenciones (García, 2008) sugirió hacer un seguimiento a 6,10 y 18 meses posteriores a la intervención, si bien no señaló las actividades a realizar.

\section{Teoría psicológica y f unciones que desempeña el psicólogo}

\section{Modelo teórico}

En cuanto a la teoría o modelo que emplearon los investigadores para sustentar la intervención, cuatro de ellos no especificaron el modelo teórico en el que enmarcaron su diseño, uno reportó haber elaborado el marco teórico y la metodología del programa de prevención bajo la perspectiva interconductual, mientras que otro partió de tres modelos teóricos: la teoría social cognitiva, la teoría de la planeación conductual y el modelo de creencias de salud.

\section{Función del psicólogo}

Las funciones que desempeñó el psicólogo en cada intervención se encontraron definidas en el estudio de Cortés, López, Alarcón y Torres (2009): medición de estatura y peso, selección de cinco alimentos naturales (verduras y frutas) y cinco alimentos industrializados (frituras, palomitas, paletas, dulces picantes y goma de mascar) para utilizarse en la intervención; elaboración de material psicológico con contenidos alusivos a las diferencias entre comer y alimentarse balanceadamente, la relación entre el comer y la salud, y la conducción de las sesiones y el análisis de resultados.

En el estudio de García (2008), tales funciones fueron la medición de estatura y el peso de padres y menores; el diseño de un taller para modificar la actitud de los padres hacia la obesidad con temas como "¿Qué es la obesidad? y "Facto- 
res ambientales y psicológicos de la obesidad", y la selección, adaptación, aplicación y evaluación de un instrumento psicométrico.

\section{DISCUSIÓN}

La revisión sistemática reportada aquí tuvo como objetivos identificar programas de prevención de obesidad infantil en México, analizar su metodología y los indicadores de cambio empleados para evaluar su efectividad e identificar la teoría psicológica y las funciones que realizan los psicólogos en dichos programas. Con base en los resultados encontrados, fue posible observar que la mayoría de los estudios incluyeron a los padres de familia, lo cual pudo haber facilitado la promoción de hábitos saludables en sus hijos, ya que estos aprenden e imitan las conductas de las personas con las que conviven diariamente; en consecuencia, incluir a los padres o a otros familiares permitiría modificar los estilos de vida poco saludables (Mercado y Vilchis, 2013; OMS, 2018; OPS, 2014; Secretaría de Salud, 2018). Asimismo, se pueden plantear alternativas de prevención para la obesidad en función del IMC, es decir, trabajar con niños y padres que padecen obesidad de una forma diferente a aquellos con un IMC "normal".

El IMC fue el indicador más utilizado para evaluar la efectividad de los programas de prevención; al respecto, los estudios de Alvirde et al. (2013) y González et al. (2014) disminuyeron el IMC en periodos de tres años y de seis meses, respectivamente; por otro lado, el trabajo de García (2008) lo logró en poco más de un mes. Tales diferencias pueden explicarse al haberse trabajado con niños que, por consiguiente, se hallaban en periodo de rápido crecimiento. Este parámetro puede fluctuar en función del sexo, la edad y el tipo de alimentación, entre otras variables, y por tanto sería recomendable que las mediciones se hicieran en periodos más cortos que hagan posible disminuir el efecto de dichas variables fisiológicas. Se sugiere que en futuras investigaciones se pongan en práctica otras medidas antropométricas (como la circunferencia de cintura y cadera, el porcentaje de grasa, la cantidad de pliegues grasos, etc.) y psicológicas (conducta alimentaria, alimentación emocional, insatisfacción corporal, autoestima, etc.).

Howard, Hopta, Krause y Or-linsky (1986) y Kopta (2003) señalan que las intervenciones de dieciséis sesiones o más pueden afectar la validez interna del estudio debido a que los participantes pueden experimentar cansancio o pérdida de interés; no obstante, Alvirde et al. (2013) y González et al. (2014) encontraron resultados favorables en el IMC pese a que sus estudios fueron de larga duración. Pueden diseñarse programas de prevención de la obesidad infantil considerando las características de su muestra, tales como edad, niveles educativo y socioeconómico, características de los padres y características sociales, ambientales y culturales, entre muchas otras (Bernaldo, Labrador, Estupiñá y Fernández, 2013).

Respecto al uso de instrumentos en los programas de prevención, solamente en un estudio se utilizó un instrumento validado y adaptado para población mexicana, con propiedades psicométricas de confiabilidad y validez aceptables. No obstante, dos estudios crearon sus propios instrumentos de medición, sin calcular ni reportar sus propiedades psicométricas, lo que resta confiabilidad a los resultados obtenidos (Hernández, Fernández y Baptista, 2010). El empleo de un instrumento o escala psicométrica válida y confiable, así como su adaptación a la muestra de estudio, disminuirá el error de medida, permitiendo conocer en qué medida los hallazgos y conclusiones del estudio son precisos.

Los resultados indican que en la prevención de la obesidad infantil han participado nutriólogos, médicos, enfermeras, trabajadores sociales y psicólogos, aunque ha habido muy escasa colaboración multidisciplinaria. Los estudios han hecho hallazgos parcialmente alentadores, aunque podrían mejorarse los resultados si se conjuntaran grupos de profesionales de la salud en los que se incluyera al psicólogo, toda vez que este profesional puede implementar técnicas de modificación de conducta para los hábitos pocos saludables de los niños y padres con o sin obesidad (Reynoso, 2014; Reynoso y Seligson, 2005).

El estudio de Safdie et al. (2013) fue el único en que se empleó una fase de seguimiento. Se sugiere que este tipo de intervención se efectúe 
al mes y a los tres meses de haber concluido el programa de prevención a fin de conocer qué conductas aprendidas muestran estabilidad en el tiempo, así como las posibles variaciones en las medidas antropométricas (Bados y García, 2009; Rothman, 2000).

Finalmente, se concluye que al ser la obesidad infantil en México un problema de salud pública en constante crecimiento, deben ponerse en práctica programas de prevención con las siguientes características: a) participación de los padres de familia o familiares cercanos a los menores; b) colaboración multidisciplinar de nutriólogos, médicos, enfermeros y psicólogos; c) medidas de tipo antropométrico (peso, talla, IMC, circunferencia de cintura-cadera, porcentaje de grasa y pliegues grasos) y psicológico (conducta alimentaria, alimentación emocional y satisfacción con la imagen corporal); d) instrumentos psicométricos validados y adaptados a población mexicana, y e) al menos dos seguimientos (al mes y a los tres meses), de tal forma que se puedan evaluar los cambios en el mediano plazo. Se recomienda que las siguientes revisiones sistemáticas empleen un mayor número de palabras claves, amplíen el periodo de búsqueda $\mathrm{y}$, de ser posible, realicen un metaanálisis de los datos obtenidos.

\section{AGRADECIMIENTOS}

Los autores de la presente investigación agradecen el presupuesto otorgado a la Dra. Adriana Amaya-Hernández (PAPIIT IA302618) y a la Dra. Mayaro Ortega-Luyando (PAPIIT IA302320).

Citación: Pérez O., A., Ortega L., M. y Amaya H., A. (2021). Programas de prevención de obesidad infantil en México: Una revisión sistemática PICO. Psicología y Salud, 31(2), 169-177. https://doi.org/10.25009/pys.v31i2.2686.

REFERENCIAS (Se indican con * los estudios incluidos en la revisión).

*Alvirde G., U., Rodríguez G., A., Henao M., S., Gómez P., F. y Aguilar S., C. (2013). Resultados de un programa comunitario de intervención en el estilo de vida en niños. Salud Pública de México, 55(3), 406-414.

Bados L., A. y García G., E. (2009). El proceso de evaluación y tratamiento. Dipòsit Digital de la Universitat de Barcelona. Recuperado de https://www.researchgate.net/publication/39033765_El_Proceso_de_evaluacion_y_tratamiento.

Bernaldo de Q., M., Labrador, F., Estupiñá, F. y Fernández A., I. (2013). La duración de los tratamientos psicológicos: diferencias entre casos de corta, media y larga duración. Universitas Psicológica, 12(1), 21-30. Recuperado de https:/www.redalyc. org/articulo.oa?id=64728729003.

*Cortés M., A., López G., M.R., Alarcón A., M.E. y Torres B., X. (2009). Behavioral strategy on nutritional education. Journal of Behavior, Health \& Social Issues, 1(1). 29-36. Recuperado de https:/www.redalyc.org/pdf/2822/282221718003.pdf.

Franco G., Á. (2012). Promoción de la salud (PS) en la globalidad. Revista de la Facultad Nacional de Salud Pública, 30(2), 193-201. Recuperado de http://www.scielo.org.co/pdf/rfnsp/v30n2/v30n2a08.pdf.

*García A., C.Y. (2008). Modificación en la actitud parental en relación con la obesidad infantil. Tesis inédita de licenciatura. México: UNAM. Recuperado de http://132.248.9.195/pd2008/0624228/Index.html.

*González H., R., Castañeda S., O., López M., C., Brito Z., O. y Sabag R., E. (2014). Intervención familiar para el manejo de sobrepeso y obesidad en escolares. Revista Médica del Instituto Mexicano del Seguro Social, 52(1), 74-77.

Hernández, R., Fernández, C. y Baptista, M.P. (2010). Metodología de la investigación. México: McGraw-Hill.

Howard, I.K., Kopta, S.M., Krause, M.S. y Or-linsky, D.E. (1986). The dose-effect relationship in psychotherapy. American Psychologist, 41(2), 159-164. Doi: 10.1037/0003-066X.41.2.159.

Instituto Mexicano para la Competitividad, A.C. (2015). Kilos de más, pesos de menos: Los costos de la obesidad en México. México: IMCO. Recuperado de http://oment.uanl.mx/kilos-de-mas-pesos-de-menos-los-costos-de-la-obesidad-en-mexico-2/.

Jensen, K.A. (2018). Seven steps to the perfect PICO search. Evidence-based nursing practice. EBSCO Health-CINAHL. Recuperado de https://www.ebsco.com/sites/g/files/nabnos 191/files/acquiadam-assets/7-Steps-to-the-Perfect-PICO-SearchWhite-Paper.pdf?_ga=2.92481434.60838456.1569789026-91600718.1569789026. 
Kopta, S. (2003). The dose-effect relationship in psychotherapy: A defining achievement for Dr. Kenneth Howard. Journal of Clinical Psychology, 59(7), 727-733. Doi: 10.1002/jclp.10167.

Landa R., E. y Arredondo P., A. (2014). Herramienta PICO para la formulación clínicamente relevante en la psicooncología basada en la evidencia. Psicooncología, 11(2-3), 259-270.

Lewis R.J., Cash, T.F., Jacobi, L. y Bubb-Lewis, C. (1997). Prejudice toward fat people: the development and validation of the antifat attitudes test. Obesity Research, 5(4), 297-307. Doi: 10.1002/j.1550-8528.1997.tb00555.x.

Lizaraso, F. (2012). Promoción de la salud: un tema pendiente. Horizonte Médico, 12(2), 6-7.

Mercado, P. y Vilchis, G. (2013). La obesidad infantil en México. Alternativas en Psicología, 17(28), 49-57.

Muñoz, F. y Arango, C. (2017). Obesidad infantil: un nuevo enfoque para su estudio. Salud Uninorte, 33(3), 492-503. Doi: 10.14482/sun.33.3.10916.

Organización Mundial de la Salud (2016). ¿Qué es la promoción de la salud? Ginebra: OMS. Recuperado de http://www.who. int/features/qa/health-promotion/es/.

Organización Mundial de la Salud (2018a). Obesidad y sobrepeso. Ginebra: OMS. Recuperado de http://www.who.int/mediacentre/factsheets/fs311/es/.

Organización Mundial de la Salud (2018b) ¿Por qué son importantes el sobrepeso y la obesidad infantiles? Ginebra: OMS. Recuperado de http://www.who.int/dietphysicalactivity/childhood_consequences/es/.

Organización Panamericana de la Salud (2014). Plan de Acción para la Prevención y Control de la Obesidad en Niños y Adolescentes. Washington, D.C.: OPS. Recuperado de http://www.paho.org/hq/index.php?option=com_contentyview=articleyi$\mathrm{d}=11373$ yltemid $=41740$ ylang $=$ es.

Ortega C., R. (2014). Costos económicos de la obesidad infantil y sus consecuencias (Editorial). Revista Médica del Instituto Mexicano del Seguro Social, 52(Supl. 1), S8-S11. Recuperado de https://www.medigraphic.com/pdfs/imss/im-2014/ ims141c.pdf.

Puente V., C.E. (2008). El modelo ecológico de Bronfenbrenner: una contribución para el estudio de la construcción cultural de la vejez en la Ciudad de México. Tesis inédita de maestría. México: UNAM. Recuperado de http://132.248.9.195/ptd2008/ diciembre/0637292/Index.html.

Reynoso E., L. (2014). Medicina conductual: Introducción. En L. Reynoso E. y A. Becerra G. (Eds.): Medicina conductual: Teoría y práctica (pp. 11-26). México: UNAM-Qartuppi.

Reynoso E., L. y Seligson, I. (2005). Psicología clínica de la salud: un enfoque conductual. México: El Manual Moderno.

Rivas R., R. y Talavera J., O. (2012). VII. Búsqueda sistemática: cómo localizar artículos para resolver una pregunta clínica. Revista Médica del Instituto Mexicano del Seguro Social, 50(1), 53-58.

Rivera, J., Hernández, M., Aguilar, C., Vadillo, F. y Murayama, C. (Eds.) (2012). Obesidad en México: Recomendaciones para una política de Estado. México: UNAM.

*Rivera, M., Priego, H. y Córdova, J. (2010). Programa educativo de alimentación y nutrición en una comunidad del Estado de Tabasco. Horizonte Sanitario, 9(3), 7-15. Doi: 10.19136/hs.a9n3.143.

Rothman, A. (2000). Toward a theory-based analysis of behavioral maintenance. Health Psychology, 19(1), 64-69. Doi: 10.1037/0278-6133.19.Suppl1.64.

*Safdie, M., Lévesque, L., González C., I., Salvo, D., Islas, A., Hernández C., S. y Rivera J., A. (2013). Promoting healthful diet and physical activity in the Mexican school system for the prevention of obesity in children. Salud Pública de México, 55(Supl. 3), 357-373.

Secretaría de Salud (2018). DGPS-Nuestros Programas. México: SSA. Recuperado de https://www.gob.mx/salud/documentos/ dgps-nuestros-programas.

Shamah, T., Cuevas, L., Rivera, J. y Hernández, M. (2016). Encuesta Nacional de Salud y Nutrición a Medio Camino 2016 (ENSANUT-MC 2016). Recuperado de https://www.gob.mx/cms/uploads/attachment/file/209093/ENSANUT.pdf.

Torrico, M.E., Santín, M.C., Andrés, M., Menéndez, S. y López, M.J. (2008). El modelo ecológico de Bronfrenbrenner como marco teórico de la psicooncología. Anales de Psicología, 18(1), 45-59. 